American Journal of Applied Sciences 5 (12): 1737-1741, 2008

ISSN 1546-9239

(C) 2008 Science Publications

\title{
Quality Evaluation Techniques of Processing the ECG Signal
}

\author{
Mikhled Alfaouri and Khaled Daqrouq \\ Department of Communication and Electronics Engineering, \\ Philadelphia University, Amman, Jordan
}

\begin{abstract}
In this research, methods to detect and evaluate the main parameters of the ECG signal are presented in order to improve its clinical properties. Two techniques are addressed in this research, Piecewise method and the Analytical Approach. These techniques are used to determine such as: percent (rms) difference (PRD), signal-noise-ratio (SNR) and the compression ratio (CR) which are used to evaluate the quality of ECG signals. The evaluation and processing of the methodology quality and the metrological point of view of ECG signal problems are subsequently obtained from detection and filtration. This technique has been successfully implemented to determine the legal accuracy rang of ECG signal for detection, filtration and compression.
\end{abstract}

Key words: Quality evaluation, ECG, PRD, CR, SNR

\section{INTRODUCTION}

In recent years, many methods of processing the Electrocardiogram (ECG) signal were reported ${ }^{[1,2]}$. Since it is difficult to get an accurate real value of the measured property of the ECG signal due to the fluctuation of the surrounding environment effects of measuring methods used, the proposed methods are well suited to the treatment of the quality measuring method to determine and provide accurate and reliable clinical information. The accuracy of the measuring method is defined as the closeness of the real value when compared to the measured property.

The difference between real value and achieved value of measured property is known as the error.

\section{MATERIALS AND METHODS}

Brief survey of error computational method: Nearly all the problems of interest of the ECG signal processing application are suffering from error and a lacking of accuracy. The authors felt that there are need to address this particular problem to situate it within the ECG signal computational. The error consists of two scalar measures. Defined as:

$$
\Delta=\mathrm{V}_{\mathrm{a}}-\mathrm{V}_{\mathrm{r}}, \delta=\left(\mathrm{V}_{\mathrm{a}}-\mathrm{V}_{\mathrm{r}}\right) / \mathrm{V}_{\mathrm{r}}
$$

where, $\Delta$ is the error, $\delta$ is the relative error, $\mathrm{V}_{\mathrm{r}}$ is the real value and $\mathrm{V}_{\mathrm{a}}$ is the achieved value. The error which repeats itself for each measurement, is called systematic error and the error which is in each measurement is different, is called random or accidental. The error related to the measurement of ECG signals (vectors) is a vector values can be represented and defined as:

$$
\Delta=\mathbf{V}_{\mathrm{a}}-\mathbf{V}_{\mathrm{r}}
$$

where, $\Delta$ can be considered as a discrete random function $^{[1]}$ :

$$
\Delta=\Delta(\mathrm{i}), \mathrm{i}=1,2, \ldots \mathrm{N}
$$

The measurement of the error can be achieved by two groups: moment and centre moment groups. These two moments are defined, respectively, as:

$$
\begin{aligned}
& \mathrm{m}_{\Delta, \mathrm{k}}=1 / \mathrm{N}\left(\Sigma(\Delta(\mathrm{i}))^{\mathrm{k}}\right) \\
& \mu_{\Delta, \mathrm{k}}=1 / \mathrm{N}\left(\Sigma\left(\Delta(\mathrm{i})-\mathrm{m}_{\Delta, 1}\right)^{\mathrm{k}}\right)
\end{aligned}
$$

depending on $\mathrm{k}$ the above moments can give several statistical parameters:

- $\mathrm{m}_{\Delta, 1}$ is $\Delta_{\mathrm{m}}$ - mean value of the error vector,

- $\mathrm{m}_{\Delta, 2}$ is $\Delta_{\mathrm{mse}}$ - mean square of the error vector,

- $\mu_{\Delta, 2}$ is $\sigma^{2}$ - the variance value of the error vector,

- $\quad\left(\mu_{\Delta, 2}\right)^{1 / 2}$ is $\sigma$ - the standard deviation of the error vector.

The error evaluation of digital signal processing methods is used to determine the quality of these

Corresponding Author: Mikhled Alfaouri, Department of Communication and Electronics Engineering, Philadelphia University, Amman, Jordan 
methods. This evaluation of the quality assists greatly in deciding how much the used method is useful. This research presents several methods of ECG signal processing such as filtration and detection. Many error analysis methods can be used to make sure that the processing method used are suiTable for ECG signal. The PRD algorithm was used to determine the deformation of the signal after filtering. The PRD algorithm measures error between signals interval. Thus the PRD algorithm provides information of how much the proposed method is useful ${ }^{[3]}$. SNR determines the signal to noise ratio. Many other algorithms are used for ECG signal compression evaluation such as CR. In this research, two ECG models are presented: piecewise linear ECG model PL-model ${ }^{[1]}$ and analytic ECG model $\mathrm{AM}^{[2]}$. The ECG model signals are used for quality evaluation of ECG processing methods because the values of these signals are previously known. This makes the evaluation easier.

Piecewise linear ECG model: In this model, evaluation and processing of the ECG signal using piecewise linear model (PL-model) is achieved by time scale and amplitude scale vectors, defined respectively as:

$$
\begin{aligned}
& \mathrm{T}=\left[0, \mathrm{~T}_{1}, \mathrm{~T}_{2}, \mathrm{~T}_{3}, \ldots, \mathrm{T}_{\mathrm{s}}\right], \\
& \mathrm{W}=\left[\mathrm{W}_{0}, \mathrm{~W}_{1}, \mathrm{~W}_{2}, \mathrm{~W}_{3}, \ldots, \mathrm{W}_{\mathrm{s}}\right] .
\end{aligned}
$$

Figure 1. Shows the graphical form of PL-model where the broken points or (characteristic points) determined by the above vectors with the same number of elements.

Where, $\mathrm{N}=5$. And the characteristic point:

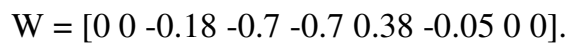

Figure 1 is generated using the algorithm of PLmodel with amplitude resolution $2^{-\mathrm{M}}$ and time resolution $2^{-\mathrm{N}}$ of the ECG model signal.

The ECG signal for different beats is shown in Fig. 2 and 3.

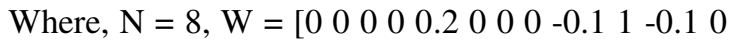
$00.280000000 .20000-0.11-0.1000 .2800000$ $0.20000-0.11-0.1000 .28000000000 .20000-0.11$ $-0.10000 .28000000 .20000-0.11-0.10000 .2800$ ]; and $\mathrm{T}=\left[\begin{array}{llllllllllll}0 & 30 & 50 & 60 & 70 & 80 & 90 & 110 & 120 & 130 & 140 & 150\end{array}\right.$ 170190210220230250260270280290310320330 340350370390410420430450460470480490510 520530540550570590610620630650660670680 690710720730740750770790810820830850860 8708808909109209309409509709901010 1024].

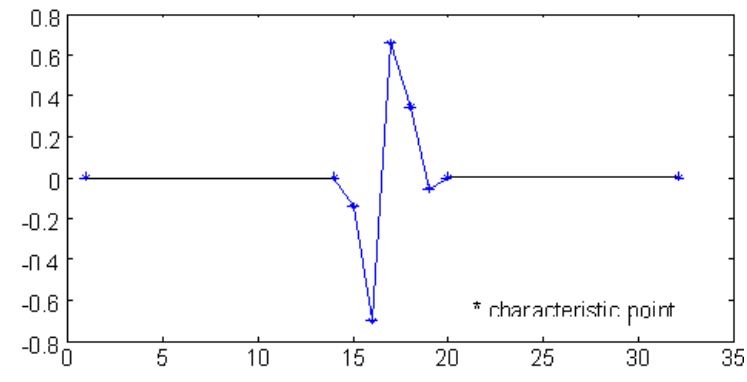

Fig. 1: The PL-model signal

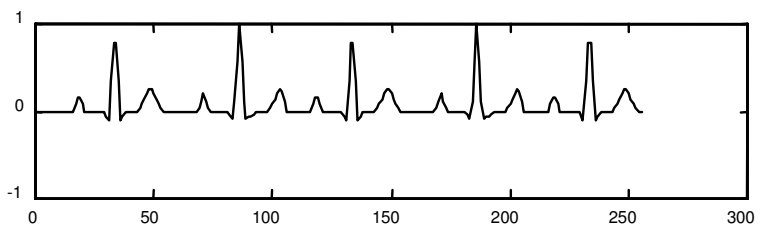

Fig. 2: The ECG model signal for different beats

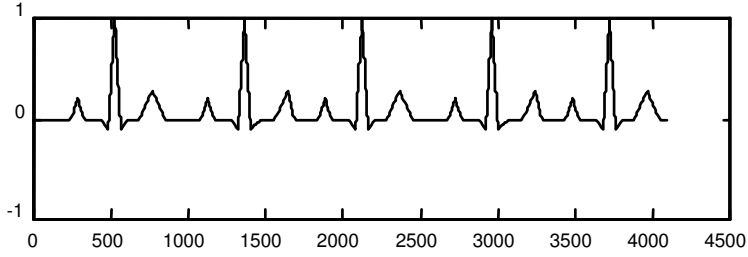

Fig. 3: The ECG model signal for different beats with $\mathrm{N}=12$, Wand $\mathrm{T}$ as in Fig. 2

with $\mathrm{N}=12$, Wand $\mathrm{T}$ as in Fig. 2.

A version of the (PL) algorithm in a detailed form:

1. Set the first value sample index $i=0$ and the piece $\mathrm{p}=1$

2. calculate $\mathrm{t}_{\mathrm{i}}=\mathrm{T}_{\mathrm{s}} / 2^{\wedge} \mathrm{N}_{* i \mathrm{i}}$,

3. calculate $\mathrm{f}_{\mathrm{i}}(\mathrm{i}+1)=((\mathrm{W}(\mathrm{p})-\mathrm{W}(\mathrm{p}-1)) /(\mathrm{T}(\mathrm{p})-\mathrm{T}(\mathrm{p}-1))) *($ $\left.\mathrm{t}_{\mathrm{i}}-\mathrm{T}(\mathrm{p}-1)\right)+\mathrm{W}(\mathrm{p}-1)$,

4. save $\mathrm{f}_{\mathrm{i}}$ as a vector,

5. $\mathrm{i}+1$, Calculate $\mathrm{t}_{\mathrm{i}}=\mathrm{T}_{\mathrm{s}} / 2^{\wedge} \mathrm{N}_{*} \mathrm{i}$,

6. if $\mathrm{t}_{\mathrm{i}}<\mathrm{T}_{\mathrm{p}}$ go to 3 if not go to 7 ,

7. $\mathrm{p}+1$,

8. if $\mathrm{t}_{\mathrm{i}}<\mathrm{T}_{\mathrm{s}}$ go to 3 if not STOP,

Simulating of ECG PL-model: PL-model represents the ECG clinical signal information at this point ${ }^{[4]}$. Next calculating the rmse of ECG original-PL, using root mean square error (rmse) to compare the original $\left(f_{\text {org }}\right)$ ECG with the model signal $\left(f_{m}\right)$ through the following Eq.

$$
\text { rmse }=1 / \mathrm{N}\left(\Sigma\left(\mathrm{f}_{\mathrm{m}}-\mathrm{f}_{\text {org }}\right)^{2}\right)^{1 / 2}
$$


Table 1: title Missing

\begin{tabular}{lc}
\hline No. of characteristic points & RMSE \\
\hline 10 & 1.1686 \\
12 & 0.1301 \\
16 & 0.2679 \\
20 & 0.3774 \\
\hline
\end{tabular}
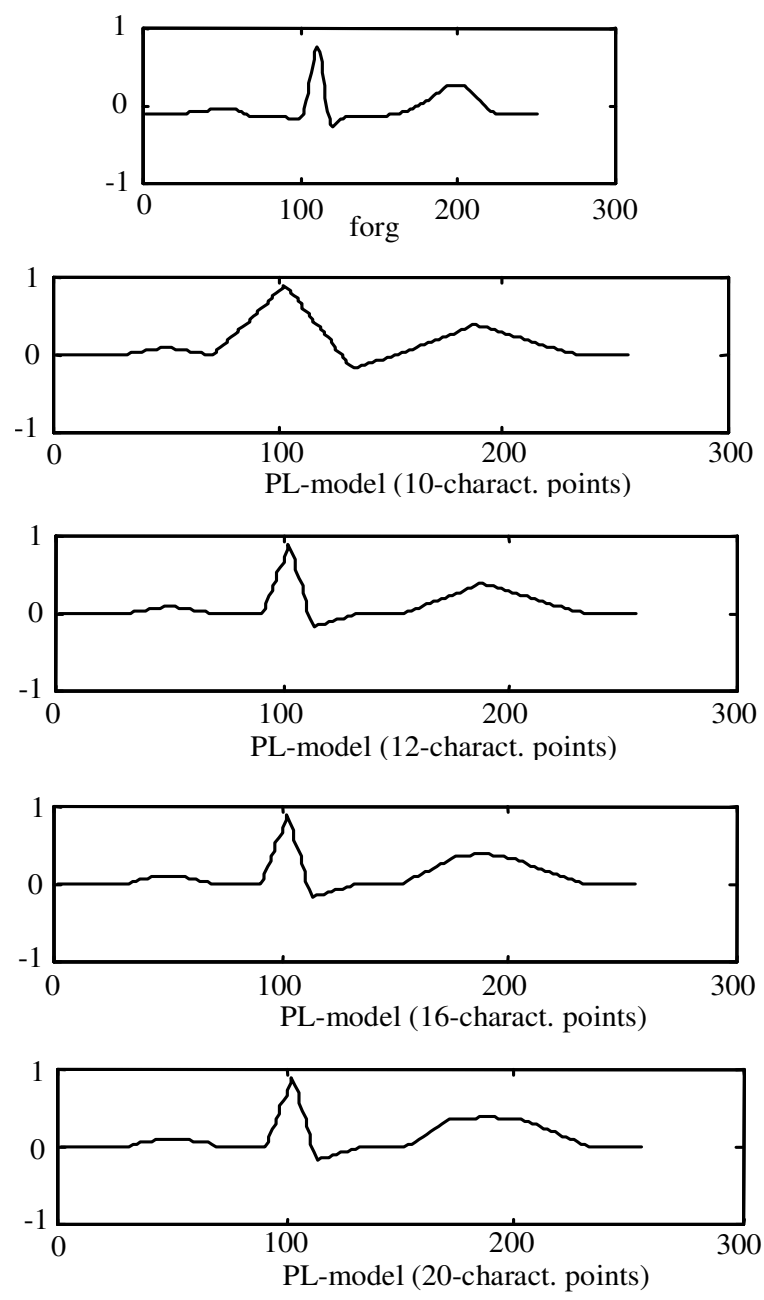

Fig. 4: The original ECG and PL-model with different numbers of characteristic points

Note that from Eq 5, increasing the number of characteristic points improves the results and guarantees good presentation of original signal. The possibility of generating PL-model with more characteristic points or less make it flexible and suiTable to represents clinical information of ECG signal as shown in both (Table 1, Fig. 4).

The ECG PL-model signal is good for metrological evaluation of many algorithms for filtration, detection and compression. PL model is used to describe filtering and detection of ECG signal parameters.
Table 2: PRD and SNR of PL-model signal filtration

\begin{tabular}{lll}
\hline Filter. of PL-model & F Noised & F Filtered \\
\hline PRD [\%] & $\ldots \ldots \ldots \ldots$. & 0.0066 \\
SNR [dB] & 30.65 & 38.764 \\
\hline
\end{tabular}
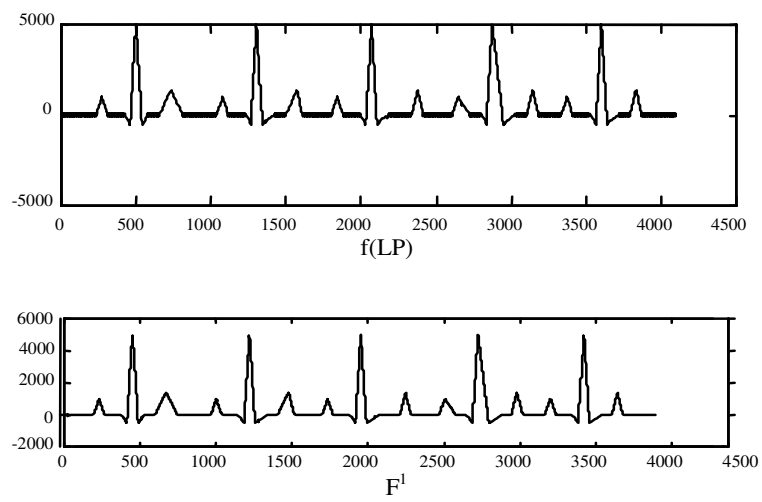

Fig. 5: ECG - PL-model filtration result

Table 1 and Fig. 4 represent the rmse comparison original ECG signal using PL-model with different numbers of characteristic points

Evaluation of filtration methods using PL-model: At this stage, ECG model signals can be created in any form needed such as evaluate the quality of filtration. This enables us to decide which algorithm is suiTable for the distinct nature of ECG signal. In this research, quality evaluation of filtering methods of the ECG PLmodel signal is determined by PRD of the deformation of the filtered signal and SNR. PL-model noise signal was added of standard deviation of $2.2 \mathrm{mV}$ [3]. PRD and SNR values of filtered signal of the PL-model are presented in (Table. 2, Fig. 5).

The results show that ECG - PL-model can be used instead of original signal to evaluate the filtration method and researchers can if they have no access to the original ECG signals.

\section{Evaluation of detection method using PL-model:} Now, the ECG model signals is available to evaluate the detection algorithms, PL model wave was created and was detected by detection algorithm as presented in $^{[4]}$, where the peak of $\mathrm{P}$ wave was detected by continuous wavelet transform (CWT) using db1 (Haar) wavelet function as the first sample after zero crossing of CWT. The onset of $\mathrm{P}$ wave was detected as the first sample of negative wave of CWT curve and the offset of $\mathrm{P}$ wave was detected as the last sample of positive wave of CWT curve as shown in Fig. 6 using the known characteristic points found in Table 1. 

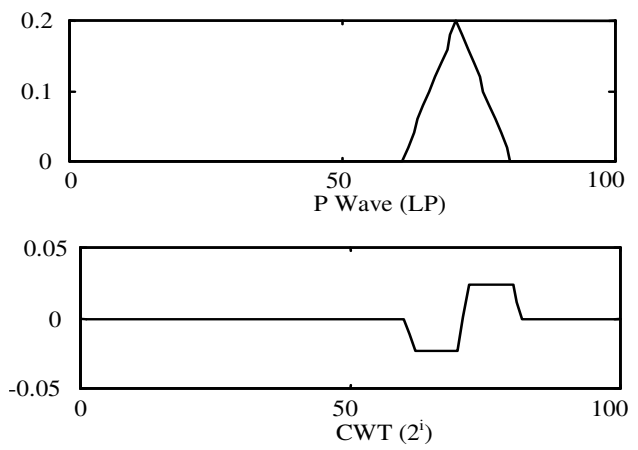

Fig. 6: The detection results of $P$ wave of PL-model. In the right side, it is presented the output of detection algorithm, where $\mathrm{i}$ is the sample number of the detection point. The detection results:

$$
\begin{array}{lll}
\text { i-P onset } & \text { i- Ppeak } & \text { i-P offset } \\
60 & 71 & 81
\end{array}
$$

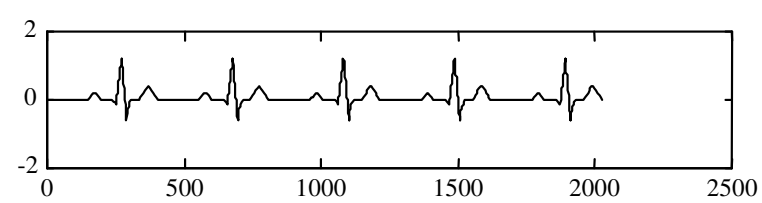

Fig. 7: The ECG model AS of few beats. The parameters were: $\mathrm{ap}=0.2 ; \mathrm{tp}=33 ; \mathrm{pq}=28$; $\mathrm{aq}=0.3 ; \mathrm{tq}=13 ;$ ar $=1.2 ;$ as $=0.6 ; \operatorname{tr} 2=17$ $\operatorname{tr} 1=19 ; \mathrm{ts}=19 ;$ at $=0.4 ; \mathrm{tt}=70 ; \mathrm{ttp}=120$; tst $=54$;

\section{RESULTS AND DISCUSSION}

Analytic ECG model signal (AM): In order to increase the accuracy of the clinical ECG signal information shown in Fig. 7, analogue normal or abnormal of signal has to be considered.

This is achieved by defining a mathematical description for each ECG fragment separately as in ${ }^{[2]}$.

P wave: $\mathrm{u}(\mathrm{t})=\left(\mathrm{A}_{\mathrm{p}} / 2\right) *\left(\sin \left(\left(2 * \mathrm{pi} * \mathrm{t} / \mathrm{t}_{\mathrm{p}}\right)+(3 * \mathrm{pi} / 2)\right)+\right), \mathrm{t} \in$ $\left.<0, t_{p}\right\rangle$ where, $A_{p}$ is $P$ wave amplitude $(0.05-0.25 \mathrm{mV})$ and $t_{p}$ is duration time of $P$ wave $(60-100 \mathrm{~ms})$.

PQ-segment: $\mathrm{u}(\mathrm{t})=0, \quad 60 \mathrm{~ms} \leq \mathrm{t} \leq 100 \mathrm{~ms}$

Q wave: $\mathrm{u}(\mathrm{t})=\left(-\mathrm{A}_{\mathrm{q}} / \mathrm{t}_{\mathrm{q}}\right) * \mathrm{t} ; \mathrm{t} \in<0, \mathrm{t}_{\mathrm{q}}>$ where $\mathrm{A}_{\mathrm{q}}$ is $\mathrm{Q}$ wave amplitude $(0.1-0.5 \mathrm{mV})$ and $\mathrm{t}_{\mathrm{q}}$ is duration time of Q wave ( $>40 \mathrm{~ms})$.

R wave: $u(t)=\left(\left(\left(A_{r}+A_{q}\right) / t_{r 1}\right) * t\right)-A_{q}, t \in<0, t_{r 1}>($ Raising fragment) where, $A_{r}$ is $R$ wave amplitude $(0.4-2.0 \mathrm{mV})$ and $t_{r 1}$ is duration time ( $\left.>40 \mathrm{~ms}\right)$.
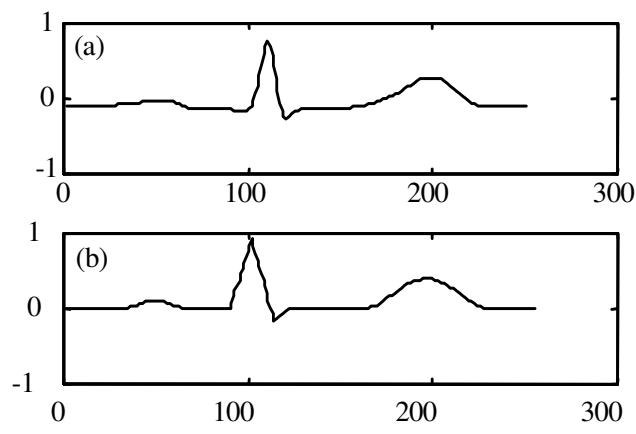

Fig. 8: a The original ECG and (b). AM

\begin{tabular}{lll} 
Table 3: & & \\
\hline AM model & F Noised & F Filterd \\
\hline PRD $[\%]$ & $\ldots \ldots \ldots .$. & 0.0130 \\
SNR $[\mathrm{dB}]$ & 30.70 & 35.857 \\
\hline
\end{tabular}

R wave: $u(t)=-\left(\left(\left(A_{r}+A_{s}\right) / t_{r 2}\right) * t\right)+A_{r}, t \in<0, t_{r 2}>$ (dropping fragment), where $\mathrm{A}_{\mathrm{s}}$ is $\mathrm{S}$ wave amplitude (0$1.0 \mathrm{mV})$ and $\mathrm{t}_{\mathrm{r} 2}$ is duration time ( $\left.>40 \mathrm{~ms}\right)$.

S wave: $\mathrm{u}(\mathrm{t})=\left(\left(\mathrm{A}_{\mathrm{s}} / \mathrm{t}_{\mathrm{s}}\right) * \mathrm{t}\right)-\mathrm{A}_{\mathrm{s}}, \mathrm{t} \epsilon<0, \mathrm{t}_{\mathrm{s}}>$ where $t_{s}$ is duration time of $S$ wave $(>60 \mathrm{~ms})$.

ST-segment: $\mathrm{u}(\mathrm{t})=0, \quad 50 \mathrm{~ms} \leq \mathrm{t} \leq 150 \mathrm{~ms}$

P wave: $\mathrm{u}(\mathrm{t})=\left(\mathrm{A}_{\mathrm{t}} / 2\right) *(\sin ((2 * \mathrm{pi} * / \mathrm{t} t)+(3 * \mathrm{pi} / 2))+1), \mathrm{t} \in$ $\left.<0, t_{t}\right\rangle$ where, $A_{t}$ is $T$ wave amplitude $(0.1-0.6 \mathrm{mV})$ and $t_{t}$ is duration time of $\mathrm{T}$ wave $(100-250 \mathrm{~ms})$.

TP-segment: $u(t)=0,210 \mathrm{~ms} \leq t \leq 470$

The sin function is used in the AM model approximation especially for $\mathrm{P}$ or $\mathrm{T}$ waves reprignal sin function. Figure 8 shows that these waves are not smooth as the orignal sin function.

This means that the AM model is slightly worse than PL-model. Henece, the PL-model with possibility of using different characteristic points number is more suiTable for clinical information presentation of ECG signal $^{[5]}$.

Table 3 presents the result of the AM model filtering with added noise shown in Fig. 9.

Comparing Table 2 with Table 3, the PRD of the filtered signal of the PL- model was less than PRD of filtered signal of the model AM and SNR is better for PL- model shown in Fig. 10. This means that ECG PLmodel is more robust to noise and more flexible to the possibility of controlling the resolution $\mathrm{N}$ of processing model signal. 

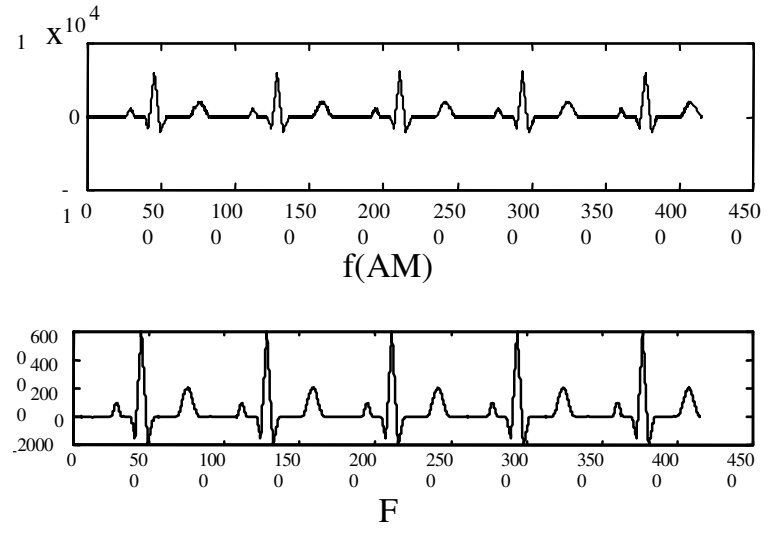

Fig. 9: ECG-AM signal filtration result
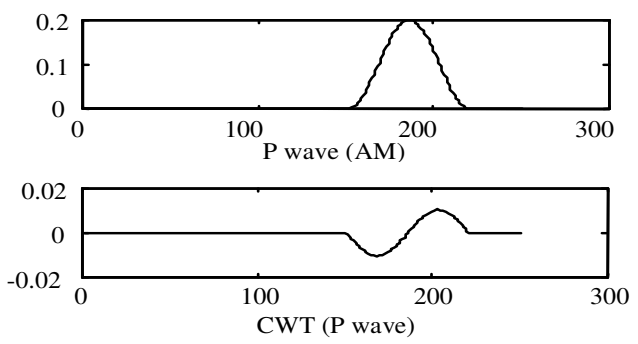

Fig. 10: The detection results of $P$ wave of model AM, on right side is presented detection results output where $i$ is the index (sample number) of detected point. The detection results:

$\begin{array}{lll}\text { i-P onset } & \text { i- Ppeak } & \text { i-P offset } \\ 150 & 186 & 221\end{array}$

\section{CONCLUSIONS}

It can be seen that evaluating the detection algorithm performance are clearly indicated in both proposed models. Compared results indicate that using the PL-model is easier to evaluate the results because of known characteristic points while with the AM model the duration time is known instead.

ECG signal has been used to recognise the most of human heart's variability, so it is very important to get parameters with high quality measurement methods.
The most important advantageous of these methods is accuracy, which should fit the level that guarantees panancea of ECG signal without losing the clinical information. The cardiology requirements demand designing many algorithms for many needs such as automatic detection of main ECG signal parameters. The detection assists greatly the cardiologist to recognise the significant deviation of the regular values of these parameters. Thus, large error in the detection of ECG signal parameters makes the algorithm not useful. Also, the filtration of ECG signal may cause large deformation of ECG signal.

It is very important to establish the legal rang of ECG apparatus accuracy to guarantee good quality of ECG apparatus manufacture. Results indicate accuracy rang of ECG signal processing methods such as detection, filtration or compression, which guides the researcher to develop his algorithm to meet the legal accuracy requirement.

Also, it is observed that the PL-model is much more pronounced for the evaluation of the detection algorithm performance.

\section{REFERENCES}

1. Jalaleddini, S., R. Strattan and W. Ceberly, 1990. ECG data compression techniques. IEEE Trans. Biomed. Eng., 37 (4): 329-342

2. Mikhled Alfaouri and Khaled Daqrouq, 2008. ECG signal denoising by wavelet transform thersholding, Am. J. Applied Sci. 5 (3): 276-281.

3. Almenar, V. and A. Albiol, 1999. A new adaptive scheme for ECG enhancement. Signal Processing 75: 253-265.

4. Mikhled Alfaouri and Khaled Daqrouq, 2007. Evaluation method of reconstruction signals using wavelet transform. Fourth International MultiConference on Systems, Signals and DevicesHammamet, Tunisia.

5. Chen, J. and S. Itoh, 1998. A wavelet transform based ECG compression method guaranteeing desired signal quality. IEEE Trans. Biomed. Eng., 45 (12). 